\title{
El traductor olvidado: Ma Yifu y el primer intento de traducir Don Quijote al chino (1905)*
}

\author{
Chen Mengyun **
}

\begin{abstract}
Resumen
Hasta hoy en día, la mayoría de los estudios cervantistas sostienen la idea de que Lin Shu (1852-1924) es el primer traductor de Don Quijote al chino (1922). Sin embargo, durante mucho tiempo, se ha ignorado a otro traductor chino, Ma Yifu (1883-1967), que tradujo Don Quijote mucho antes que Lin Shu, en 1905. De hecho, en 2008, el estudioso japonés Tarumoto Teruo ya había descubierto su traducción, publicada en el Semanario de la independencia, en 1913. Pero la idea errónea es tan popular y arraigada que continúa en muchos estudios hasta la actualidad. Por otro lado, Ma Yifu es mucho menos conocido que Lin Shu, incluso para los chinos, lo que hace más difícil corregir esta idea equivocada. El presente trabajo pretende presentar una visión general de la vida de Ma Yifu y de su traducción de Don Quijote, «La biografía del señor Ji». De manera intencionada, hemos querido prestar especial atención a los factores extratextuales de la traducción, los cuales configuran su contexto sociocultural de creación y de recepción, intentando recuperar el proceso poco conocido de la recepción del Quijote en China.
\end{abstract}

Palabras clave: Don Quijote en China; Ma Yifu; «La biografía del señor Ji»; factores extratextuales de la traducción.

Title: The Forgotten Translator: Ma Yifu and his Chinese Translation of Don Quixote (1905)

\begin{abstract}
Until today, most Cervantist studies support the idea that Lin Shu (1852-1924) is the first translator of Don Quixote into Chinese. However, we had ignored another Chinese translator for a long time, Ma Yifu (1883-1967) who translated Don Quixote, long before Lin

* El presente trabajo ha sido realizado gracias a la beca concedida por China Scholarship Council.

** Universidad de Granada. mengyun@correo.ugr.es / ORCID iD: https://orcid.org/0000-00026964-7940
\end{abstract}


Shu, in 1905. In fact, in 2008, the Japanese intellectual, Tarumoto Teruo had already discovered his translation published in Independence weekly in 1913. But the misconception is so popular and ingrained that is still widely used in many studies to the present. On the other hand, Lin Shu is much more popular than Ma Yifu in China, which makes more difficult to correct this entrenched misconception. The present work focuses on presenting a general vision of the life of Ma Yifu and his translation of Don Quixote, «The biography of Mr. Ji». We desire to pay special attention to the extratextual factors of translation, which configures its sociocultural context of creation and reception, trying to fill the little-known area of receiving Don Quixote in China.

Keywords: Don Quixote in China; Ma Yifu; «Mr. Ji’s biography»; Extratextual Factors of Translation.

\section{Cómo citar este artículo / Citation}

Chen Mengyun. 2021. «El traductor olvidado: Ma Yifu y el primer intento de traducir Don Quijote al chino(1905)».Anales Cervantinos 53: 157-171.https://doi.org/10.3989/anacervantinos.2021.006

\section{INTRODUCCIÓN}

Sobre la primera traducción china de Don Quijote de la Mancha, es creencia general que apareció en 1922, realizada por Lin Shu (1852-1924) y Chen Jialin (1800-?) y titulada La biografía del caballero loco (o El héroe mágico según las traducciones) $)^{1}$. Se trata de la traducción de la primera parte. Así lo indica Liu Xiaopei en su artículo «Cervantes en China»:

Una vez publicada la primera parte del Quijote, son sus primeras traducciones las versiones inglesa y francesa editadas en 1612 y 1614, respectivamente. En cuanto a la versión china, apareció en 1922, cotraducida por Lin Shu y Chen Jialin y titulada El héroe mágico. Es una traducción en lengua clásica china, pero traducida de manera reducida de otra versión extranjera (Liu Xiaopei 1991, 320).

La misma opinión mantienen Zhao Zhenjiang y Teng Wei $(2016,172)$ :

La primera versión en chino de Don Quijote, [...], se conoció bajo el título de La historia de un caballero loco, corresponde a la primera parte de la novela, y fue realizada en el año 1922 por Lin Shu (1852-1924) y su ayudante Chen Jialin a partir de una versión en inglés.

Chen Guoen y Zhao Hongying $(2014,66)$ sostienen la misma idea:

\section{1. 魔侠传}


The first scientific literary review of Don Quixote was made by Zhou Zuoren in his History of European Literature, published in 1918 [...] In 1922, Lin Shu, co-editing with Chen Jialin, adapted the translation of the first volume of Don Quixote into ancient Chinese, renaming it Legend of a Magic Knight, which was published by Shanghai Commercial Press. This was the first Chinese version of Don Quixote.

Aparte de los estudios citados anteriormente, prestigiosos hispanistas chinos, como Chen Zhongyi $(2014,143)$ y Chen Kaixian $(2005,228)$, por ejemplo, creen también que la primera versión china es la de Lin Shu y Chen Jialin.

Sin embargo, la primera traducción china de Don Quijote no es La biografía del caballero loco, sino «La biografía del señor Ji» ${ }^{2}$, realizada por Ma Yifu en 1905, y publicada en el Semanario de la independencia ${ }^{3}$, números 21 y 22, con el seudónimo de Pi He [被褐], en el año 1913. Se trataba de una traducción del primer capítulo completo y una parte del segundo de la primera parte de Don Quijote.

El primer estudioso que puso al descubierto esta circunstancia fue el sinólogo japonés Tarumoto Teruo (1948-), que planteó esta idea por primera vez en su artículo 最初の漢訳「ドン・キホーテ」 [《La primera traducción china de Don Quijote»] (Tarumoto 2008, 1-6). Luego, algunos estudiosos chinos hicieron referencia a esta idea. Sin embargo, bastantes hispanistas la habían presentado de forma errónea. Error que continúa en muchos estudios hasta la actualidad.

Aquí habría que mencionar el proyecto de Francisco López Fabra de traducir el capítulo XLII de la segunda parte de Don Quijote a cien lenguas. En él se incluía una traducción al chino de este capítulo, realizada por Juan Licópolis Marzal y corregida por Wên JüehSain, Lin YüTsai y Wên LanPu, en 1873 y, por lo tanto, anterior a las de Ma Yifu y de Lin Shu. Pero no fue publicada hasta 1941 en Barcelona (Imp. Casa Provincial de Caridad): De los primeros consejos que dio Don Quijote a Sancho antes que se fuese a gobernar la Insula. Texto original y traducciones [en diversas lenguas]. La obra, que formaba parte de las Publicaciones cervantinas patrocinadas por Juan Sedó Peris-Mencheta (Torres 2005, 85), y consiguientemente, la traducción del capítulo correspondiente, no pudo ser conocida en China hasta después de su publicación. Entonces, si hablamos de las traducciones al chino de Don Quijote de acuerdo con las fechas de publicación, la de Ma Yifu, más antigua que la traducción de La biografía del caballero loco de Lin Shu, debe ser considerada como la primera, que también se convierte en la primera traducción publicada en chino de las obras literarias españolas ${ }^{4}$. Aunque esta tra-

2. 稽先生传. Todas las traducciones del chino son mías.

3. 独立周报.

4. Según Deng Jitian $(2012,281)$, se pensaba que la primera era 西班牙宫闱琐语 publicada en 1915. La traducción del título en español sería Anecdotario de la corte española. Pero se desconocen su original y su autor. 
ducción de Don Quijote solo se publicó en el periódico, tiene una gran importancia para la historia del intercambio chino-español, y merece la pena estudiarla más profundamente. En el presente trabajo, basándonos en las teorías propuestas por Nord (1991) sobre los factores extratextuales, y en las de la manipulación de Lefevere (2017), y partiendo de las perspectivas histórica y sociocultural, trataremos de analizar problemas como quién es el traductor, la procedencia del texto original, dónde y cuándo se realizó y publicó la traducción, con qué intención, el mecenazgo de la traducción, la recepción y la presentación de la traducción.

\section{El CASO DE MA Yifu}

Ma Yifu (1883-1967), nacido en Shaoxing, provincia de Zhejiang, fue pensador, poeta y calígrafo chino. También se le considera representante de la llamada primera generación de neoconfucionistas. En el año 1898, participó en el Examen del Distrito que era la primera escala en el sistema de examen imperial chino, y obtuvo la mejor calificación (Teng Fu 2004, 36). Este año también es vital para China. Después de la primera guerra sino-japonesa, los intelectuales chinos, dirigidos por Kang Youwei (1858-1927) y Liang Qichao (1873-1929), iniciaron la Reforma de los Cien Días (11 de junio-21 de septiembre de 1898). Aunque al final fracasó, este intento de reformar el país causó un gran impacto entre los intelectuales, despertando su interés en estudiar las disciplinas occidentales para encontrar la vía de salvar la nación china. En el año 1899, Ma Yifu y su amigo Xie Wuliang (1884-1964) llegaron a Shanghái para estudiar inglés, francés y otras disciplinas de la cultura occidental. Para despertar la nación china, ellos y Ma Junwu (1881-1940) fundaron la revista mensual El mundo de la traducción del siglo $X X$ en Shanghái en 1901, traduciendo y presentando la política, la filosofía, la sociología y otras ideas avanzadas occidentales (Teng Fu 2004, 39). Pero esta revista solo publicó seis números y se suspendió a causa de que Ma Yifu y Xie Wuliang se fueron al extranjero (Ma Yifu 2013b, 8).

Después de la Reforma de los Cien Días, estudiar en el extranjero se convirtió en una moda de aquel momento y por ello, una oleada de chinos se fue a otros países. Ma Yifu también quería estudiar en Japón, pero no lo logró (Teng Fu 2004, 39). Hasta que en el año 1903, el gobierno Qing publicó un anuncio para contratar a un trabajador para la duodécima Exposición Universal, y Ma Yifu fue contratado sirviendo como secretario de la comisión de supervisión de los estudiantes chinos que pertenecía a la embajada del gobierno Qing en Estados Unidos. Se fue de Shanghái a finales de mayo, y llegó a San Luis a principios de julio (Ma Yifu 2013b, 6).

Al principio de su estancia en San Luis, se cortó su coleta y cambió su túnica china por el traje occidental para mostrar su voluntad de estudiar en el extranjero. Durante esta época, Ma Yifu leyó abundantes libros occidentales 
sobre literatura, filosofía, economía política, sociología, etc. Tenía el hábito de escribir en su diario, donde se mencionan muchos maestros occidentales: Spencer, Hegel, Darwin, Rousseau, Kant, Dante, Karl Marx, Shakespeare, Byron, etc. Y no solo leyó sus obras, sino que tradujo varias de ellas al chino (Ma Yifu 2013a, 1-56).

Por un lado, se inspiró en los conocimientos occidentales, por otro, $\mathrm{Ma}$ Yifu también vio los males que afligían a la sociedad estadounidense, y sintió la discriminación racial que tenían los occidentales contra los chinos. Según sus diarios, durante su estancia en Estados Unidos muy frecuentemente se sentía amargado y triste, no tenía otra solución que escribir a sus amigos, Xie Wuliang y Ma Junwu, para expresar sus emociones. Analizando su experiencia de la vida y sus diarios, hay tres causas principales que pueden explicar este estado anímico: las desgracias de su vida, el clima, y su autoestima nacional. Por lo que se refiere a la primera, cuando Ma Yifu solo tenía 11 años, perdió a su madre y después del Examen de Distrito, su padre y su esposa también murieron. Estas desgracias siempre le hicieron sentir soledad y dolor. En relación con la segunda, hay que señalar que Ma Yifu no se acostumbraba al clima de los Estados Unidos y siempre estaba enfermo. Las condiciones de su vivienda eran pésimas. En sus diarios, son frecuentes expresiones como «morir de calor» o «morir de frío». En cuanto a la tercera, y más importante, el joven Ma Yifu tenía una fuerte autoestima nacional y no podía aguantar las discriminaciones sufridas y la ignorancia que tenía la federación estudiantil china en los Estados Unidos. Por eso, en aquella tierra americana apenas tenía amigos (Teng Fu 2004, 44).

Ejemplos de esta última causa son fáciles de encontrar en sus diarios: el 25 de octubre de 1903 expresaba su indignación y tristeza al escuchar el discurso sobre si se podría desmembrar China entre los estudiantes de dos universidades estadounidenses. Ma Yifu escribió que «Quienes tienen gana de desmembrar China ya consideran cuidadosamente y discuten mucho, pero la mayoría de nuestra gente todavía está en la ignorancia. ¡Qué desafortunado!» (Ma Yifu 2013a, 33).

Con estas emociones, aunque durante su estancia en Estados Unidos leyó abundantes libros occidentales, no había ninguna escuela que influyera demasiado en él. Por el contrario, cambió sus ideas sobre la ciencia occidental, creyendo que no todo lo occidental era bueno. Así lo dejó escrito en su diario:

La belleza abstracta de la ciencia y la filosofía forman la belleza concreta del país. Los europeos y americanos actuales podemos decir que pueden fundar el país bello. Pero en cuanto a la sociedad bella todavía quedan defectos por vencer (Ma 2013a, 44).

$\mathrm{Y}$ todo eso también influye en su posterior "regreso" al conocimiento clásico chino.

El 6 de mayo de 1904 Ma Yifu salió de Estados Unidos, con la intención de visitar a sus amigos en Japón e iniciar sus estudios en dicho país. A finales 
de 1904 regresó a China. Como no encontró buenas ofertas de trabajo, vivió como un ermitaño en Zhenjiang y Hangzhou, sucesivamente. Se concentró en el estudio académico, sintetizando los conocimientos occidentales logrados y haciendo diversas comparaciones entre la ciencia occidental y la ciencia china. En el año 1905, cuando estaba en Zhenjiang, tradujo Don Quijote al chino (Ma Yifu 2013b, 10).

En los primeros años de su regreso a China, prestó atención no solo a la sabiduría occidental, sino también a la oriental. Pero poco a poco abandonó los conocimientos occidentales. No encontramos ninguna obra suya escrita después de 1912 en la cual se mencione la ciencia occidental, y se dedicó totalmente a la investigación de la sabiduría china, el budismo y el confucionismo, porque, en su opinión, la cultura occidental era una cultura de "materia" y no cuidaba la formación del espíritu. Para salvar a su nación era necesario encontrar un método desde el origen del problema, educando así el espíritu de sus compatriotas con la cultura tradicional china.

En cuanto a las causas sobre el cambio de su trayectoria investigadora, Teng $\mathrm{Fu}(2004,46)$ señala que, aunque leyó muchos libros occidentales durante su estancia en San Luis, le faltó el estudio sistemático de la sabiduría occidental, y con intención de encontrar una solución para salvar a su patria de la situación desesperada lo antes posible, solo prestó atención al aspecto práctico de la ciencia occidental. Por lo tanto, su idea sobre la cultura occidental no fue suficientemente objetiva. Eso también es una limitación típica de los chinos de aquella época. Desde la Primera Guerra del Opio (1939-1942), China siempre había estado invadida, lo que despertó dudas y sospechas sobre la cultura tradicional en los intelectuales. Como consecuencia, en los movimientos revolucionarios antiimperialistas y antifeudales, se achacaron los problemas sociales a la cultura que crearon los antepasados, criticándola en su totalidad. Eso causó dolor y decepción a Ma Yifu, quien era un defensor de la cultura tradicional china, y al mismo tiempo, sus ideas eran inaceptables por su carácter social. En la época, cuando casi todos los intelectuales chinos intentaron aprender los saberes y experiencias occidentales, a causa de su duda de la ciencia occidental y su psicología pesimista, motivada esta última por las desgracias familiares y los trastornos sociales, Ma Yifu eligió finalmente la cultura tradicional china, y se fue convirtiendo poco a poco en un maestro del neoconfucionismo y en un ermitaño particular entre los intelectuales chinos del momento.

\section{LA REVISTA JIANG $S H U^{5}$ Y LA PROCEDENCIA DEL ORIGINAL DE «LA BIOGRAFÍA DEL SEÑOR JI»}

Ya sabemos que la fecha de la traducción de «La biografía del señor Ji» es el año 1905. Nos surge otro problema: ¿de dónde procede el original? De

5. 江苏.

ANALES CERVANTINOS, VOL. LIII, pp. 157-171, 2021, ISSN: 0569-9878, e-ISSN: 1988-8325

https://doi.org/10.3989/anacervantinos.2021.006 
acuerdo con las palabras que encabeza la traducción: «Mavslated from Servantes lnly» (Ma Yifu 1913a, 61) ${ }^{6}$, el texto original sería una versión inglesa. En los primeros años del siglo XX, la literatura occidental estaba recién introducida en China. En 1904, en toda China se publicaron un total de 40 traducciones de obras literarias extranjeras, y la mayoría fueron obras de literatura de género, procedentes de Francia, Inglaterra y Japón (Deng Jitian 2012, 504). En ese momento, Cervantes, Don Quijote, y la literatura española, no eran conocidos. En el caso de los hermanos Zhou, que eran los pioneros más importantes de la literatura moderna china, no leyeron Don Quijote hasta 1908, cuando estudiaban en Japón (Qian Liqun 2007, 165 166). Entonces es poco probable que Ma Yifu lograra la traducción inglesa del Quijote en China por aquel momento. De acuerdo con su biografía, se puede deducir que tenía más posibilidad de lograrla en Japón o en Estados Unidos. En sus diarios, en los que anotaba detalles de su vida, sobre todo de su estudio, y mencionaba a numerosos escritores occidentales y sus obras, no encontramos ningún dato relacionado con Cervantes ni con Don Quijote. Por eso, tenemos que suponer que no consiguió el libro en Estados Unidos, sino en Japón.

Antes de explicar la relación entre Ma Yifu, Don Quijote y Japón, tenemos que presentar primero una revista mensual llamada Jiang Shu, que fue fundada en Tokio el 27 de abril de 1904 por la asociación de paisanos de la provincia de Jiangshu, la cual estaba formada por los estudiantes y algunos comerciantes de dicha provincia en Japón. Era una revista revolucionaria en chino, y tenía como propósito «hablar de la corrupción» (Luo Jialun 1983, 4), presentando las doctrinas sociopolíticas de la burguesía occidental y propagando la revolución contra el imperio Qing. Asimismo contenía una sección de literatura. Esta revista estaba dirigida a intelectuales y estudiantes chinos, y no solo se publicó en Tokio, Kobe y Yokohama, sino que también tenía la sede central en Shanghái y puntos de venta en otras ciudades chinas.

Antes de que Ma Yifu tradujera Don Quijote al chino, el 15 de mayo de 1904 esta revista había presentado a Cervantes y Don Quijote a los lectores chinos en un artículo titulado «Los maestros mundiales». En nuestra opinión, es el primer artículo en chino en el que se menciona a Cervantes y su inmortal obra:

\footnotetext{
沙文第斯 Cervantes 西班牙人。一千五百四十七年，卒一千六百十六 年。著唐贵孙脱 Don Quixote 小说不追前轨, 莫步后尘, 曼然称杰作 (Luo Jialun 1983, 2011).
}

Cervantes, de España, nació en 1547, y murió en 1616. Escribió Don Quijote. Esta novela no sigue el camino trillado y tiene un estilo original, $\mathrm{y}$ es tan primorosa que vale la pena de ser llamada una obra inmortal.

6. «Mavslated from Servantes lnly» son erratas de imprenta del artículo original. Debe ser «Translated from Cervantes July 1905». 
Estas palabras nos proporcionan dos datos útiles: primero, en 1904 Don Quijote había recibido la atención, la lectura y alta consideración de algunos estudiantes chinos que estaban en Japón; segundo, la versión de Don Quijote que se mencionó en la revista Jiang Shu era inglesa. De hecho, en Japón, la primera traducción de Don Quijote había aparecido en el año 1887 . Y luego, una revista japonesa publicó un listado de obras maestras de los autores más estimados del mundo, en el que figuraba Don Quijote. Y en 1893, se publicó otra versión japonesa, dentro de la serie Sekai bunkô [Biblioteca mundial], traducida por Matsui Shôyô (松居松葉，1870-1933). Era la primera traducción japonesa de la primera parte de Don Quijote. Y gracias a ella, se elevaron las imágenes de Cervantes y de su obra en Japón, colocándola como una de las obras clásicas más grandes en la historia de la literatura universal ${ }^{7}$.

Obviamente, la descripción de Don Quijote en la revista Jiang Shu siguió las ideas populares de esta obra en Japón, admirando su valor literario.

Cuando Ma Yifu abandonó Estados Unidos, tuvo una estancia corta en China y luego fue a Japón. En aquel momento, sus amigos íntimos Xie Wuliang y Ma Junwu también se encontraban allí.

Xie Wuliang era colaborador del periódico $S u B a o$, y propagó la revolución contra el imperio Qing. En 1903, el gobierno Qing ordenó embargar la redacción de $S u$ Bao y detener a las personas relacionadas, y Xie Wuliang se fugó a Japón con el redactor jefe de dicho periódico, Zhang Shizhao. En Tokio, estudió japonés, inglés y alemán (Peng Hua 2009, 135).

En cuanto a Ma Junwu, en 1901 fue a Yokohama, Japón. Dos años después, ingresó en la Universidad Imperial de Tokio, estudiando química. Durante su estancia en Japón, escribió artículos para el Periódico del nuevo pueblo ${ }^{8}$, y luego conoció a Sun Zhongshan (1866-1925), a quien siguió y participó junto con él en la revolución democrática, y también fue uno de los fundadores del Periódico del pueblo 9 .

Xie Wuliang y Ma Junwu eran revolucionarios democráticos contrarios al imperio Qing. A principios del siglo XX vivían en Tokio, y siempre participaron en las actividades sociopolíticas y escribieron artículos periodísticos. Y cuidaban ansiosamente el destino de su patria. Por todo eso, debieron conocer bien la revista Jiang Shu, que también se fundó y distribuyó en Tokio y tenía la misma ideología política que ellos. Es probable, entonces, que leyeran el artículo «Los maestros mundiales», publicado en Jiang Shu, y conocieran Don Quijote; e incluso que hubieran leído esta obra antes de leer dicho artículo. Como hemos mencionado, en el Japón de aquella época, el valor literario del Quijote ya se había reconocido. Es lógico, pues, que esta obra también se conociera entre los chinos que estudiaban allí. Además, por medio del correo,

7. Goikoetxea Lobo, Leire. 2015. La Traducción de la literatura española en el Japón de la era Meiji. Trabajo de fin de máster, Máster en estudios de Asia Oriental, Universidad de Salamanca, España, p. 26.

8. 新民从报.

9. 民报. 
en aquel momento, las librerías japonesas podrían comprar los libros originales desde otros países por encargos de los lectores. Así, estas librerías japonesas servían como puente entre los intelectuales chinos y la cultura occidental. De esta manera, por ejemplo, el escritor Lu Xun (1881-1936) compró la versión original alemana del Quijote en 1908 (Qian Liqun 2007, 165-166).

Cuando Ma Yifu estaba en Japón, estudió alemán y japonés con Xie Wuliang y Ma Junwu, al mismo tiempo estudió la literatura, el arte y la filosofía occidental, y de vez en cuando tradujo libros occidentales. Es posible que, gracias a estos dos amigos, Ma Yifu conociera a Cervantes y Don Quijote en Japón y comprara la versión inglesa y la llevara a China, y luego la tradujera unos meses después, cuando todavía tenía interés en esta novela.

\section{El SEMANARIO DE LA INDEPENDENCIA}

Antes de analizar la intención de la publicación de «La biografía del señor Ji», es conveniente conocer algo acerca de su mecenas, el Semanario de la independencia. Se fundó en Shanghái en septiembre de 1912, y dejó de publicarse en julio de 1913. Los redactores jefes fueron Zhang Shizhao (1881-1973) y Wang Zhongqi (1880-1913). Su propósito fue publicar artículos objetivos y neutrales sin implicarse en los debates entre partidos, y presentar a los lectores la verdad. Se publicaron principalmente los comentarios políticos o los comentarios de los asuntos actuales cada domingo. Y tenía más de diez puntos de distribución en Shanghái, y cuarenta y cuatro en otros lugares, cubriendo así gran parte de China. Sobre el origen de los artículos publicados, excepto los de los redactores jefes, casi todos procedían de lectores voluntarios.

Pero poco después, debido a la publicación de algunos artículos con cierta ligereza, y sin la más mínima preocupación por la veracidad, el Semanario de la independencia recibió muchas críticas sociales, y en noviembre de 1912 Zhang Shizhao dejó de ser redactor jefe de este periódico y marchó a Pekín por invitación del entonces presidente de la República de China, Yuan Shikai (1859-1916). Pero como descubrió que tenía ambición autoritaria, participó en la segunda revolución contra su gobierno. Después, al enterarse de que Wang Zhongqi había aceptado el soborno de Yuan Shikai, no volvió a tomar parte en ninguna actividad de dicho semanario (Long Minxian 2004, 67).

Así, el Semanario de la independencia tuvo dos etapas: antes del año 1913, Zhang Shizhao publicó uno o varios artículos en cada número. Podemos decir que, en esta etapa, el principal contenido del Semanario de la independencia eran los comentarios de Zhang Shizhao sobre la situación social y política. Y no se publicó ninguna novela.

La segunda etapa comenzó a partir del decimoquinto número (el primer número del año 1913). Se añadieron la sección de novela y otros contenidos literarios. Al mismo tiempo, se redujeron los comentarios políticos. La causa directa muy posible fue que después de que Yuan Shikai controlara el poder 
político, se ocupó de que los periódicos y las opiniones públicas no pusieran en peligro su autoridad, y así ordenó controlar estrictamente los periódicos y las revistas para dirigir la orientación de las opiniones públicas.

\title{
5. LA PUBLICACIÓN DE «LA BIOGRAFÍA DEL SEÑOR JI»
}

«La biografía del señor Ji» se publicó en los números 21 y 22 del Semanario de la independencia, el 23 de febrero y el 2 de marzo de 1913. Pero, de hecho, desde 1908 Ma Yifu apenas publicó artículos en periódicos o revistas. También rechazó las invitaciones de los editores (Ma Yifu 2013b, 13-15). Fue dejando de investigar la sabiduría occidental, concentrándose en el estudio de la sinología. Y durante este tiempo, siempre se sentía pesimista por los desprecios que sufrían los frutos culturales de los antepasados, por la corrupción de la moralidad de la sociedad y por la tiranía de los gobernantes. Después de la Revolución de Xinhai (10 de octubre de 1911-12 de febrero de 1912), Ma Yifu ya se había convertido por completo en un defensor de la cultura tradicional china (Teng Fu 2004, 75-77). Era imposible que sus ideas recibieran muchos apoyos en aquel momento, cuando casi todos tenían pasión por estudiar los conocimientos occidentales. Claro que también Ma Yifu lo sabía, por eso prefería esconderse en su propio mundo.

Entonces, ¿por qué su traducción, que había realizado unos años antes, apareció de repente en el periódico?

Antes del texto de esta traducción, podemos leer las palabras del editor que la preceden, esclarecedoras en este sentido:

\section{血海花魂记因作者染恙，尚未脱稿。本期特賡刊此篇以饷阅者。本文 为西文中最著名之作。被褐先生又以檏茂淋漓之笔译之。为自有译本 小说以来所未有。阅者幸勿忽视。 \\ 译西班牙率望之集 Mavslated from Servantes lnly 1905 (Ma Yifu 1913a, 61).}

\begin{abstract}
Como el autor de La historia del mar de sangre y del alma de flor [Xuehai huahun Ji] todavía está enfermo, no alcanza a realizarla. Entonces, en este número, publicamos especialmente este folletín para los lectores, que es de la obra más famosa occidental. El señor Pi He la tradujo con fluidez y mucha emoción. Esta traducción es la mejor que nunca han visto. Los lectores tienen suerte y no lo ignoran. Traducido de la obra de Cervantes, julio de 1905 .
\end{abstract}

El autor de La historia del mar de sangre y del alma de flor es el redactor jefe mencionado, Wang Zhongqi, con el seudónimo de Tianmiu Shen [天谬生]. Alrededor del año 1912, Wang escribió a su amigo Ma Yifu para invitarlo a tomar parte en la fundación de este periódico. Ma Yifu era un conservador de la cultura tradicional china; sin embargo, la inclinación social y el propósito del periódico era publicar conocimientos nuevos. Por 
ello, Ma pensó que no era apropiado para este periódico y rechazó la invitación de su amigo. Pero a causa de la enfermedad de Wang Zhongqi y por la salida de Zhang Shizhao, efectivamente el Semanario de la independencia no tenía material suficiente para seguir publicando. Entonces, Ma Yifu le envió a Wang Zhongqi algunos trabajos que había escrito o traducido unos años antes (Ma Yifu 2013b, 17) y, aunque no hay documentos concretos para certificarlo, podría deducirse que entre ellos estaba «La biografía del señor Ji» que tradujo en 1905. Estos artículos se publicaron en el Semanario de la independencia, números 21 y 22, el 23 de febrero y el 2 de marzo de 1913, respectivamente. Estos son: «Palabras por las callejas» ${ }^{10}$ y «La biografía del señor Ji» (Ma Yifu 1913a, 61-63); y «Continuación del bosque de sentido» ${ }^{11} \mathrm{y}$ «La biografía del señor Ji (continuación)» (Ma Yifu 1913b, 77-78).

Sabemos que en 1905, cuando vivía en Zhenjiang, Ma Yifu ya había intentado traducir Don Quijote al chino. A partir de 1913 se había concentrado en el estudio de la sabiduría tradicional china y en el budismo. Frente a la tendencia social de estudiar lo occidental, prefería ser un "ermitaño" en la época cambiante. Además, como «La biografía del señor Ji» se interrumpió de repente, suponemos que Ma Yifu no habría establecido ningún plan de realizarla, era una simple práctica traductológica. Así pues, por lo que se refiere al traductor, no tuvo intención inicial de publicarla. Quizás, sin la enfermedad de su amigo, sin el apuro del Semanario de la independencia, o si Ma Yifu hubiera rechazado la petición de su amigo otra vez, no se habría publicado esta primera traducción china de Don Quijote.

Por otra parte, Wang Zhongqi invitó a Ma Yifu a escribir artículos para la revista sin ninguna intención concreta de introducir Don Quijote. En conclusión, ni por la parte del mecenas ni por la del traductor existía intención especial de presentar completa esta obra de Cervantes. Podemos decir que su publicación fue debida al azar.

$\mathrm{Y}$ en cuanto a la recepción de los lectores, poca gente mencionó esta traducción. En principio, hasta donde sabemos, «La biografía del señor Ji» no tuvo influencia obvia en la literatura china. Esta podría ser la causa por la que los estudiosos ignoraron esta traducción hasta ahora.

\section{LAS CARACTERÍSTICAS DE LA TRADUCCIÓN DE «LA BIOGRAFÍA DEL SEÑOR JI»}

Cada obra traducida se realiza en un contexto concreto, y también está limitada por él. «La biografía del señor Ji» se creó en una época donde toda-

10. 巷语.

11. 意林续抄. 
vía no se había formulado la teoría moderna de traducción. Por lo demás, su texto original es desconocido. Por eso, no intentamos criticar su calidad de traducción de acuerdo con los principios de la traductología moderna. No obstante, para conocerla más, merece la pena identificar objetivamente las características generales de esta traducción.

«La biografía del señor Ji» contiene el primer capítulo y una parte del segundo de la primera parte de Don Quijote, hasta la conversación entre don Quijote y las dos mozas en la venta. Tiene cinco páginas en total. Sus características se pueden resumir en los siguientes puntos capitales:

1. Es una traducción parcial e indirecta, basada en una versión inglesa.

2. Tiene las características del pianwen (la prosa paralela china), utilizando frases paralelas (Ejemplo 1); la lengua utilizada en el texto traducido es wenyan (el chino clásico). Aunque Lin Shu también tradujo La biografía del caballero loco en wenyan, influido por el Movimiento de Nueva Cultura, no rechazó en absoluto el uso de palabras o expresiones en baihua (el chino vernáculo). En comparación, el wenyan usado en «La biografía del señor Ji» es más tradicional y antiguo (Ejemplo 2). En gran medida, es un resultado inevitable causado por la limitación del contexto sociocultural.

Ejemplo 1:

Limpias, pues, sus armas, hecho del morrión celada, puesto nombre a su rocín y confirmándose a sí mismo... (Cervantes 2004, 7).

由是, 甲贯既新, 宝剑既硎, 骏马既鸣, 名字既成 (Ma Yifu 1913a, 62).

Ejemplo 2:

... tiempo vendrá en que las vuestras señorías me manden y yo obedezca, y el valor de mi brazo descubra el deseo que tengo de serviros (Cervantes 2004, 7).

二子幸而役之, 某将感激承命 (Ma Yifu 1913b, 78).

异日尔二人命我, 我即出其武技 (Lin Shu 1922, 7).

3. Se caracteriza por la traducción libre. En sentido estricto, fue una adaptación parcial. Ma Yifu añadió sus ideas en el texto y eliminó algunas frases originales. Por ejemplo, en el fragmento en el que don Quijote pasa toda la noche en la venta con la celada puesta, Ma Yifu comentó:

自古未闻憌而寝者，有之，自稽叔始 (1913b, 78).

Nunca me he enterado de que alguien duerme con la celada puesta, si hay, don Quijote es el primero. 
4. Se caracteriza por la estrategia domesticadora ${ }^{12}$. Así, tradujo «el señor castellano» como «太守» ${ }^{13}$. Y frente a los elementos culturales específicos, Ma Yifu no solo los tradujo al chino, sino que también conservó las palabras originales y las explicó en chino entre corchetes. Por ejemplo:

\section{迎天女邚娜娜 aurona ${ }^{14}$ (晓之神) 来降人间 (Ma Yifu 1913b, 77).}

Habían saludado la venida de la aurora (diosa del amanecer) a nuestro mundo.

$\mathrm{Y}$ algunas traducciones de dichos elementos son tan precisas que combinan la pronunciación y el sentido original de los elementos y la característica tradicional china. Por ejemplo, tradujo el caballo del Cid, «Babieca», como 《白鼻䮐》 [bái bí guā] ${ }^{15}$; el caballo de Alejandro, «Bucephalus», como 《不死驊骝》 [bù sǐ huá liú] ${ }^{16} \mathrm{y}$ 《Dulcinea del Toboso» ${ }^{17}$ como 《東施杜蓮》 [dōng shī dù lián]. En la historia china, 《東施》 es una mujer fea que intenta imitar a la belleza, Xi Shi [西施 ${ }^{18}$. Según estos ejemplos, ya podemos ver la habilidad excelente de Ma Yifu en el inglés y en la traducción.

5. Ma Yifu añadió un total de nueve notas entre paréntesis en el texto para explicar los elementos culturales específicos como: «castellano», «knight», «Hércules», etc.

6. Otro aspecto que debemos señalar es que en esta traducción existen algunas erratas de imprenta de las palabras inglesas. Por ejemplo, «Don Ruisote» (Don Quixote), «Mavslated from Servantes Inly» (translated from Cervantes July 1905), «avtaeus» (antaeus), «Aldvnza Dopvnzo» (Aldonza Lorenzo), «Serville» (Sevilla), etc. De eso se podría deducir la edición de mala calidad del Semanario de la independencia.

\section{CONCLUSIÓN}

De acuerdo con la fecha de publicación, «La biografía del señor Ji» de Ma Yifu debe ser considerada como el primer intento de traducción de Don Quijote al chino. Fue realizada en 1905 a partir de una traducción inglesa, y

12. Lawrence Venuti plasmó en su obra The Translator's Invisibility: A History of Translation (1995) los términos de 'domesticación' y 'extranjerización' como estrategias traslativas opuestas. Con la estrategia domesticadora, se reducen los elementos extranjeros al incorporar términos, expresiones y estructuras gramaticales habituales en la lengua meta para provocar en el lector una sensación de naturalidad y fluidez. En cambio, la extranjerización ayuda a promover la diversidad cultural y supone un reto a los valores estéticos de la cultura meta.

13. Un burócrata de la China imperial.

14. «Aurona» es una errata en el texto original.

15. En el chino clásico, 《䮐》 se refiere al caballero amarillo con boca negra.

16. 《驊骝》, en la leyenda china, se refiere al corcel alazán del rey Mu de la dinastía Zhou.

17. Se imprimió erróneamente como «Tobvso del zlurcinea» en el original.

18. Xi Shi es una de las reconocidas Cuatro Bellezas de la antigua China. 
publicada en 1913 en el Seminario de la independencia. La procedencia del original de «La biografía del señor Ji» podría ser Japón. Es una simple práctica traductológica. El traductor no tenía ninguna intención de popularizar Don Quijote en China y se publicó por casualidad. Aunque en sentido estricto es una adaptación parcial, en el contexto de su creación tiene virtudes altamente positivas, sobre todo en la traducción de los elementos culturales específicos. «La biografía del señor Ji», como el primer intento de traducir Don Quijote al chino, es testigo de la historia del intercambio literario chino-español antes de La biografía del caballero loco de Lin Shu, y no se debe ser ignorado.

\section{BIBLIOGRAFÍA CITADA}

Cervantes Saavedra, Miguel de. 2004. Don Quijote de la Mancha. Barcelona: Galaxia Gutenberg.

Chen Guoen y Zhao Hongying. 2014. "The Spread and Reception of Don Quixote in China». Advances in Literary Study 2: 66-73.

Chen Kaixian. 2005. «La recepción de Don Quijote en China». Boletín de la Biblioteca de Menéndez Pelayo 81: 227-239.

Chen Zhongyi. 2014. Sai wan ti si xueshushi yanjiu [Estudio sobre la historia de estudios cervantinos]. Nanjing: Editorial de Yilin.

Deng Jitian. 2012. Zhongguo xiandai wenxue chuban pingtai [La plataforma de edición de la literatura moderna china]. Shanghái: Editorial de Literatura y Arte de Shanghái.

Lefevere, André. 2017. Translation, Rewriting, and the Manipulation of Literary Fame. Londres: Routledge.

Lin Shu. 1922. Mo xia zhuan [La biografia del caballero loco]. Shanghái: La Prensa Comercial.

Liu Xiaopei. 1991. «Cervantes en China». En Actas del II Coloquio Internacional de la Asociación de Cervantistas (Alcalá de Henares, 6-9 nov. 1989), 319-325. Barcelona: Anthropos.

Long Minxian. 2004. «Zhang Shizhao yu duli zhoubao» [«Zhang Shizhao y el Semanario de la independencia»]. Chuban kexue [La ciencia de publicación] 4: 67-68.

Luo Jialun, ed. 1983. «Sijie weiren» [«Los maestros mundiales»]. En Zhonghua minguo shiliao congbian Jiang Shu [Colección de los documentos históricos de la República de China Jiang Shu], 2010-2014. Taipei: Comisión de Recopilación de la Historia de Kuming-tang.

Ma Yifu. 1913a. «Ji xianshen zhuan» [«La biografía del señor Ji»]. Duli zhoubao [El Semanario de la independencia] 21: 61-63.

Ma Yifu. 1913b. «Ji xianshen zhuan (xu)» [«La biografía del señor Ji (continuación)»]. Duli zhoubao [El Semanario de la independencia] 22: 77-78.

Ma Yifu. 2013a. Ma Yifu quanji: Riji [La obra completa de Ma Yifu: Diarios], ed. Guang Wu. Hangzhou: Editorial de los Libros Antiguos de Zhejiang.

Ma Yifu. 2013b. Ma Yifu quanji: Fulu [La obra completa de Ma Yifu: Apéndice], ed. Guang Wu. Hangzhou: Editorial de los Libros Antiguos de Zhejiang.

Nord, Christiane. 1991. Text analysis in translation. Theory, Methodology, and Didactic Application of a Model for Translation-Oriented Text Analysis. Ámsterdam/Atlanta: Rodopi. 
Peng Hua. 2009. «Xie Wuliang nianpu» [«Cronología de Xie Wuliang»]. En Ru zang luntan [El foro de los Cánones Confucianos], ed. Dagang Shu, 134-135. Chengdu: Editorial de la Universidad de Sichuan.

Qian Liqun. 2007. Fengfu de tongku [Los dolores abundantes]. Pekín: Editorial de la Universidad de Pekín.

Tarumoto Teruo. 2008. 最初の漢訳「ドン.キホーテ」[《La primera traducción en chino de Don Quijote»]. En 清末小説から(通訊) [Shinmatsu shōsetsu Kara] 88: 1-6.

Teng Fu. 2004. Ma Yifu zhuan [La biografia de Ma Yifu]. Hangzhou: Editorial de Hangzhou.

Torres Santo Domingo, Marta. 2005. «El primer facsímile del Quijote: la aventura editorial de Francisco López Fabra (1871-879)». En El Quijote en las bibliotecas universitarias españolas, 73-96. Ciudad Real: Empresa Pública "Don Quijote de la Mancha 2005".

Venuti, Lawrence. 1995. The Translator's Invisibility: A History of Translation. London: Routledge.

Zhao Zhenjiang y Teng Wei. 2016. «La traducción y difusión de Don Quijote en China». En Don Quijote en los cinco continentes, ed. Hans Christian Hagedorn, 171-186. Cuenca: Ediciones de la Universidad de Castilla-La Mancha.

Recibido: 14 de abril de 2020

Aceptado: 14 de julio de 2020 
